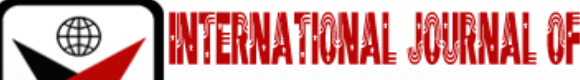

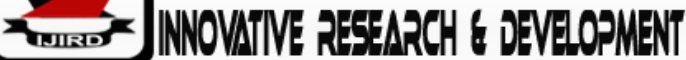

ISSN 2278-0211 (Online)

\section{One-Step Rational Integrators Method for Solving Initial Value Problems (IVPs) in Ordinary Differential Equations}

Ebhohimen Fidelis
Lecturer, Department of Mathematics,
Ambrose Alli University, Ekpoma, Edo State, Nigeria
Osemwegie U. I.
Assistant Lecturer, Department of Mathematics,
College of Education, Ekiadolo-Benin, Edo State, Nigeria
Anetor Osemenkhian
Assistant Lecturer, Department of Mathematics,
Ambrose Alli University, Ekpoma, Edo State, Nigeria

\section{Abstract:}

In this paper work we designed one - Step Rational Integrators (OSRIs) of order $2 k-1$ method for solving initial value problems (IVPS) in Ordinary Differential Equations (ODEs). The method was implemented and compared with other existing methods to ascertain the level of performance, in terms of results produced from solving some initial value problems. Problems involving modeling of real-life situation in an ordinary differential equation were handled by our method.

Keywords: One-Step, rational integrators, stability, consistency and convergence

\section{Introduction}

This research work is concerned with the determination of solution to a class of problem in Ordinary Differential Equations (ODEs). There have been three major directions in which research have been channeled, namely: the modeling and simulation group, the abstract and classical analysis group and the computerized group, Aashikpelokhai, 2014.

The modeling and simulation group looks at real-life problems, Obodi, 2001 observed the rate at which a given real-life problem is changing with respect to an observable single variable and then goes ahead to model and simulate an ODE based on the observations. Enright and Pryce, 1987 compiled a collection of modeled ODE from various industries which today are used in testing the power and direction of computerized method highlighted in group three.

Our second group: Abstract and Classical Analysis group considered an ODE with specific unknown parameters or unknown coefficients of the ODE terms, subjects the ODE to certain constraints and then obtain conditions or forms under which the coefficients or parameters According to (Aashikpelokhai, 2014), Most current research work in classical mathematics to which ODE belongs have been in existence since $1642 \mathrm{AD}$; that is, in the past 362 years. It is not therefore a surprise that this area is richest and even tending towards abstraction, Aghajani and Moradifan, 2007.

The researchers in the computerized group concern themselves with the development, analysis, testing implementation of methods to solve problems in ODE, Aashikpelokhai, 1991. This group handles effectively problems whose solutions cannot be established with ease through abstract and classical methods but to evaluate them without computational methods becomes extremely difficult. These are the main reasons why in today's world of ODE, emphasis is drifting to computerized mathematics whenever one is handling most crucial problems in this area. We implement Aashikpelokhai, 1991, rational integrator formula using a matlab software to establish our result. Examples of most recent work in this area include Onianwa and Aashikpelokhai, 2010; Elakhe and Aashikpelokhai, 2013; and Ukpebor and Aashikpelokhai, 2014; Ebhohimen and Anetor 2017.

The problem for this research paper is to find a numerical solution to the IVP which is represented by,

$y^{\prime}=f(x, y), \quad y\left(x_{0}\right)=y_{0} a \leq x \leq b$

Where $\mathrm{f}(\mathrm{x}, \mathrm{y})$ is defined and continuous in a region $\mathrm{D} \subset[\mathrm{a}, \mathrm{b}]$,

However, this research paper is therefore to design and implement One -Step rational integrators method that can cope effectively well with such problems. Our aims in this research paper is to solve problem represented by (1.1) where $\mathrm{f}(\mathrm{x}, \mathrm{y})$ must satisfy a Lipchitz condition with respect to $\mathrm{y}$.

Definition 1.1: A one-step method is said to be A-STABLE if when applied to the test equation $y^{1}=\lambda y$ with $\operatorname{Re}(\lambda)<0$, it gives, $\mathrm{y}_{\mathrm{n}+1}=s(\bar{h}) y_{n}$ with the stability function $\mathrm{S}(\overline{\mathrm{h}})$ satisfying, 
$|s(\bar{h})|<1$ for all $\operatorname{Re}(\bar{h})<0, \bar{h}=\lambda h$

Definition 1.2. A given One-Step Method is said to be L-Stable if it is A-Stable and in

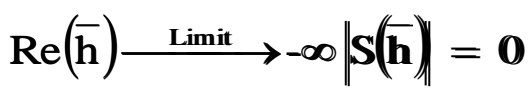

\section{The Derivation of the One - Step Rational Integrator Method}

$y_{n+1}=\frac{\sum_{i=0}^{k-1} p_{i} X_{n+1}^{i}}{1+\sum_{i=0}^{k} q_{i} X_{n+1}^{i}}=\frac{\sum_{i=0}^{2} p_{i} X_{n+1}^{i}}{1+\sum_{i=0}^{3} q_{i} X_{n+1}^{i}}$

Let $\mathrm{X}_{\mathrm{n}}=\mathrm{nh}$

Expanding $\mathrm{y}_{\mathrm{n}+1}$ about $\mathrm{x}_{\mathrm{n}}$ in Taylor series expansion the integrator 1.2 becomes

$\mathrm{y}_{\mathrm{n}+1}=\sum_{\mathrm{i}=1}^{\mathrm{k}-1} \mathrm{P}_{\mathrm{ih}} \mathrm{i}^{\mathrm{i}(\mathrm{n}+1)^{\mathrm{i}}} \sum_{\mathrm{i}=0}^{\infty}(-1)^{\mathrm{i}}\left(\sum_{\mathrm{i}=1}^{\mathrm{k}} \mathrm{P}_{\mathrm{i} h^{\mathrm{i}}(\mathrm{n}+1)^{\mathrm{i}}}\right)^{i}$

The Taylor series expansion for theoretical solution $y_{n+1}$ about the point $x_{n}$ is given as:

$y_{\left(X_{n+1}\right)}=\sum_{i=1}^{\infty} \frac{h^{i}}{i !} y_{\left(X_{n}\right)}$

Assumed that $y_{\left(X_{n}\right)}=y_{n}$ and $y_{\left(X_{n}\right)}^{(m)}=y_{n}{ }^{m} 1.5$

Hence a direct result of application of equation (1.4) makes the first $2 \mathrm{k}$ terms to be written as:

$y_{\left(X_{n+1}\right)}=\sum_{i=0}^{2 k-1} \frac{h^{i} y_{n}^{(i)}}{i !}$

Equating the terms in powers of $\mathrm{h}$ we get:

$h^{0} \rightarrow p_{0}=y_{n}$

$h^{1} \rightarrow p_{1}=-\frac{h y_{n}^{(1)}}{x_{n+1}}+y_{n} q_{1} 1.8$

Next, we combine equation 1.7 and 1.8 as we equate the terms in $h^{2}$ to get

$p_{2}=\frac{h y_{n}^{(1)}}{2 ! X_{n+1}}+\frac{h y_{n}^{(1)}}{X_{n+1}} q_{1}+y_{n} q_{2}$

If we continue with this procedure of equating corresponding coefficients in powers of $h$ and writing the resulting equations as a combination of all the previous equations we get:

$p_{j}=\frac{h^{j+1-i} y_{n}^{(j+1-i)} q_{i-1}}{(j+1-i) ! X_{n+1}^{j+1-i}}+y_{n} q_{j}, \mathrm{j}=1(1) \mathrm{k}-1$

Where $q_{0}=1$

$$
y_{n}^{0} \quad=y_{n}
$$

We are still left with $\mathrm{k}$ equation to cope with. Equation 1.10 gives the result for $\mathrm{p}_{\mathrm{i}}^{\text {'s }} \mathrm{s}$ as being fully dependent on some or on all of the parameters qi's.

Let consider the case of $\mathrm{k}=3$, one can have the remaining equations written exclusively in terms of only unknown parameters.

$\mathrm{q}_{\mathrm{i}, \mathrm{i}}=1(1) \mathrm{k}$

Applying the process, we already used above, we write the equation resulting from equating a given power of $\mathrm{h}$ as a combination of the immediate $\mathrm{k}$ preceding equations, the following results were obtained.

$$
\text { For } \mathrm{h}^{\mathrm{k}} \rightarrow \sum_{j=1}^{k} \frac{h^{k-j} y_{n}^{(k-j)} q_{i}}{(k-j) ! x_{n+1}^{k-j}}=-\frac{h^{k} y_{n}^{(k)} q_{i}}{(k) ! x_{n+1}^{k+1}}
$$

For $\mathrm{h}^{\mathrm{k}+1} \rightarrow \sum_{j=1}^{k} \frac{h^{k+1-j} y_{n}^{(k-j)} q_{j}}{(k+1-j) ! x_{n+1}^{k+1-j}}=-\frac{h^{k+1} y_{n}^{(k+1)}}{(k+1) ! x_{n+1}^{k+1}}$

$$
\text { For } \mathrm{h}^{\mathrm{k}+2} \rightarrow \sum_{j=1}^{k} \frac{h^{k+2-j} y_{n}^{(k-j)} q_{j}}{(k+2-j) ! x_{n+1}^{k+2-j}}=-\frac{h^{k+2} y_{n}^{(k+2)}}{(k+2) ! x_{n+1}^{k+2}}
$$

For $\mathrm{h}^{2 \mathrm{k}-3} \rightarrow \sum_{j=1}^{k} \frac{h^{2 k-3-j} y_{n}^{(2 k-3-j)} q_{j}}{(2 k-3-j) ! x_{n+1}^{2 k-3-j}}=-\frac{h^{2 k-3} y_{n}^{(2 k-3)}}{(2 k-3) ! x_{n+1}^{2 k-3}}$ 
For $\mathrm{h}^{2 \mathrm{k}-2} \rightarrow \sum_{j=1}^{k} \frac{h^{2 k-2-j} y_{n}^{(2 k-2-j)} q_{j}}{(2 k-2-j) ! x_{n+1}^{k-2-j}}=-\frac{h^{2 k-2} y_{n}^{(2 k-2)}}{(2 k-2) ! x_{n+1}^{2 k-2}}$

$$
\text { For } \mathrm{h}^{2 \mathrm{k}-1} \rightarrow \sum_{j=1}^{k} \frac{h^{2 k-1-j} y_{n}^{(2 k-2-j)} q_{j}}{(2 k-1-j) ! x_{n+1}^{k-1-j}}=-\frac{h^{2 k-1} y_{n}^{(2 k-1)}}{(2 k-2) ! x_{n+1}^{2 k-1}}
$$

To obtain an expression for solving for the integrator parameters $q_{i}, i=1(1) k$, we express $k$ equations namely equation $1.14-1.19$, in a matrix form as shown below:

$\mathrm{Sq}=\mathrm{b}$

Where

$s_{i j}=\frac{h^{2 k-i-j} y_{n}^{(2 k-i-j)}}{(2 k-i-j) ! x_{n+1}^{2 k-i-j}}, i, j=1(1) k$

defines the entries of the matrix $S$,

$$
q=\left[\begin{array}{c}
q_{1} \\
q_{2} \\
q_{3} \\
\cdot \\
\cdot \\
\cdot \\
q_{n}
\end{array}\right] 1.22
$$

and

$$
b_{i}=\frac{h^{2 k-i} y_{n}^{(2 k-i)}}{(2 k-i) ! x_{n+1}^{2 k-i}}
$$

$q_{0}$ and $y_{n}^{(0)}$ are given by 1.11 and 1.12 respectively.

\section{Local Truncation Error}

The $(2 \mathrm{k}-1)^{\text {th }}$ term is the series expansion for $y_{n+1}$ in the case of an arbitrary positive integer $\mathrm{k}$ is expressed as a combination of the k proceeding terms in the series to yield:

$\mathrm{x}_{\mathrm{n}+1}^{2 \mathrm{k}}=\sum_{i=1}^{k} \frac{h^{i} y_{n}^{(2 k-i)} q_{i}}{(2 k-i) ! \mathrm{x}_{\mathrm{n}+1}^{2 \mathrm{k}-\mathrm{i}}}$

Which is the same thing as:

$-\mathrm{x}_{\mathrm{n}+1}^{2 \mathrm{k}}=\mathrm{b} \cdot \mathrm{q}$

Where the vectors $\mathrm{b}$ and $\mathrm{q}$ are given in equation 1.22 and 1.23above. By neglecting the term in $h^{2 k+1}$, we have the truncation error given by:

$\mathrm{T}_{\mathrm{n}+1}=\frac{\mathrm{h}^{2 \mathrm{k}-\mathrm{i} \mathrm{y}_{\mathrm{n}}(2 \mathrm{k})}}{(2 \mathrm{k}) !}-x_{n+1}^{2 k} b \cdot q 1.26$

\section{The Convergence of the Scheme}

According to Fatunla (1982), a One - Step rational integrators scheme is given as:

$\mathrm{y}_{\mathrm{n}+1}=\mathrm{y}_{\mathrm{n}}+\mathrm{h}$ ? $\left(\mathrm{x}_{\mathrm{n}}, \mathrm{y}_{\mathrm{n}}, \mathrm{h}\right)$

is said to be convergent if for arbitrary initial vector $\mathrm{y}_{0}$ and an arbitrary point $\mathrm{x} \epsilon$ [a,b], the global error is given as:

$\mathrm{e}_{\mathrm{n}+1}=\mathrm{y}_{\mathrm{n}+1}-y\left(\mathrm{x}_{\mathrm{n}+1}\right)$

satisfies the following relationship

$\mathrm{h} \stackrel{\text { limit }}{\longrightarrow} 0 \max e_{n}=0$

provided $\mathrm{x}$ is always a mesh point.

\section{Theorem: Convergence and Consistency}

One - step rational integrator if

$y_{n+1}=\frac{\sum_{i=0}^{k-1} p_{i} X_{n+1}^{i}}{1+\sum_{i=0}^{k} q_{i} X_{n+1}^{i}}$

is convergent.

Proof

A One - step rational integrator of the form 
$\mathrm{y}_{\mathrm{n}+1}=\mathrm{y}_{\mathrm{n}}+\mathrm{h} \emptyset\left(\mathrm{x}_{\mathrm{n}}, \mathrm{y}_{\mathrm{n}}, \mathrm{h}\right)$

where ? $\left(\mathrm{x}_{\mathrm{n}}, \mathrm{y}_{\mathrm{n}}, \mathrm{h}\right)$ is the increment function and $\mathrm{h}$ is the meshsize adopted in the subinterval [ $\left.\mathrm{x}_{\mathrm{n}}, \mathrm{x}_{\mathrm{n}+1}\right]$,is convergent if and only if it is consistent [Lambert (33)].To establish the convergence of the One - step integrators formula, we have to show that the integrator is consistent as noted in Lambert (1978).

From equation $1.6 \mathrm{P}_{0}=y_{n}$ for every $\mathrm{k}$ which when adopted in the expression fory $\mathrm{n}_{\mathrm{1}}$ give the above yields:

$y_{n+1}=\frac{\sum_{i=0}^{2} p_{i} X_{n+1}^{i}}{1+\sum_{i=1}^{3} q_{i} X_{n+1}^{i}}$

byre-arranging 1.2 we obtain

$y_{n+1}-y_{n}=\frac{\sum_{i=0}^{2}\left(p_{i}-q_{i}\right) X_{n+1}^{i}}{1+\sum_{i=1}^{3} q_{i} X_{n+1}^{i}}$

$\frac{y_{n+1}-y_{n}}{h}=\phi\left(\mathrm{x}_{\mathrm{n}}, \mathrm{y}_{\mathrm{n}}, \mathrm{h}\right)$

where $\phi$ is the increment function.

This leads us to,

$\phi\left(\mathrm{x}_{\mathrm{n}}, \mathrm{y}_{\mathrm{n}}, 0\right)=\left(\mathrm{p}_{1}-\mathrm{q}_{1} \mathrm{y}_{\mathrm{n}}\right)(\mathrm{n}+1) \quad$ i.e. $n+1=\frac{x_{n+1}}{h}$

$\phi\left(\mathrm{x}_{\mathrm{n}}, \mathrm{y}_{\mathrm{n}}, 0\right)=\left(\mathrm{p}_{1}-\mathrm{q}_{1} \mathrm{y}_{\mathrm{n}}\right) \cdot \frac{x_{n+1}}{h}$

But,

$$
p_{i}=\sum_{j-i}^{i} \frac{h^{i+1-j}\left(y_{n}\right)^{i+1-j} q_{j-1}}{(i+1-j) !\left(X_{n+1}\right)^{i+1-j}}+y_{n} q_{i}
$$

$p_{i}=\frac{h y_{n}^{(1)}}{1 ! x_{n+1}}+y_{n} q_{1}$

$\mathrm{p}_{1}-\mathrm{y}_{\mathrm{n}} \mathrm{q}_{1}=\frac{h y_{n}^{(1)}}{1 ! x_{n+1}}$

putting equation 1.36 into 1.34

$\phi\left(\mathrm{x}_{\mathrm{n}}, \mathrm{y}_{\mathrm{n}}, 0\right)=\frac{h y_{n}^{(1)}}{x_{n+1}} \cdot \frac{x_{n+1}}{h}$

$\phi\left(\mathrm{x}_{\mathrm{n}}, \mathrm{y}_{\mathrm{n}}, 0\right)=y_{n}^{(1)}=\mathrm{f}\left(\mathrm{x}_{\mathrm{n}}, \mathrm{y}_{\mathrm{n}}\right)=\mathrm{f}(\mathrm{x}, \mathrm{y})$

Hence, we conclude that the One-Step rational integrators are consistent with the initial value problem (ivp).

\section{The Stability of Our Scheme}

Considering $q_{i}{ }^{\prime}$ s and $p_{i}{ }^{\prime}$ s as function $\mathrm{h}$ and we obtain:

$\mathrm{q}_{\mathrm{i}}(\mathrm{h}, \mathrm{k})=\frac{(-1)^{i}(2 \mathrm{k}-1-\mathrm{i})\left(\begin{array}{c}\mathrm{k} \\ \mathrm{i}\end{array}\right) \mathrm{h}^{\mathrm{i}}}{(2 \mathrm{k}-1) ! \mathrm{x}_{\mathrm{n}+1}^{\mathrm{i}}}, \mathrm{i}=0(1) \mathrm{k}$

$\mathrm{P}_{\mathrm{i}}(\mathrm{h}, \mathrm{k})=\frac{(2 \mathrm{k}-1-\mathrm{i}) !\left(\begin{array}{c}\mathrm{k}-1 \\ \mathrm{i}\end{array}\right) \mathrm{h}^{\mathrm{i}}}{(2 \mathrm{k}-1) ! \mathrm{x}_{\mathrm{n}+1}^{\mathrm{i}}+1} y_{n}, \mathrm{i}=0(1) \mathrm{k}-1$

Consequently we have;

$\mathrm{q}_{\mathrm{i}}(\mathrm{h}, \mathrm{k})=\mathrm{q}_{\mathrm{i}}$ and $\mathrm{P}_{\mathrm{i}}(\mathrm{h}, \mathrm{k})=\mathrm{P}_{\mathrm{i}}$

Then

$\mathrm{q}_{\mathrm{i}} \mathrm{n}_{\mathrm{n}+1}^{\mathrm{i}}=\frac{(-1)^{i}(2 \mathrm{k}-1-\mathrm{i})\left(\begin{array}{c}\mathrm{k} \\ \mathrm{i}\end{array}\right) \mathrm{h}^{\mathrm{i}}}{(2 \mathrm{k}-1) !}, \mathrm{i}=0(1) \mathrm{k}$

$\mathrm{P}_{\mathrm{i}} \mathrm{X}_{\mathrm{n}+1}^{\mathrm{i}}=\frac{(2 \mathrm{k}-1-\mathrm{i}) !\left(\begin{array}{c}\mathrm{k}-1 \\ \mathrm{i}\end{array}\right) \mathrm{h}^{\mathrm{i}}}{(2 \mathrm{k}-1) !} y_{n}, \mathrm{i}=0(1) \mathrm{k}-1$

Given an arbitrary positive integer $\mathrm{k}$, applying One-Step rational integrators to equation $\mathrm{y}^{1}=\lambda \mathrm{y}$, we obtain the stability expression:

$$
\frac{y_{n+1}}{y_{n}}=\frac{\sum_{i-0}^{k-1}(2 k-1-i) !\left(\begin{array}{l}
k-1 \\
i
\end{array}\right) h^{i}}{\sum_{i-0}^{k}(-1)^{i}(2 k-1-i) !\left(\begin{array}{l}
k \\
i
\end{array}\right) h^{i}}
$$

where $\left(\begin{array}{l}k \\ i\end{array}\right)=\frac{k^{m}}{1 !\left(k^{m}-i\right) !}, k^{m}=\mathrm{k}$ or $\mathrm{k}-1$

The stability function $\mathrm{s}(\mathrm{h})$ of the One- Step rational integrators for arbitrary positive integer k is therefore given by:

$\mathrm{s}(\mathrm{h})=\frac{y_{n+1}}{y_{n}}$ 


\section{Numerical Computations and Results}

Problem 1: Aashikpelokhai (1991) and Fatunla (1983)

$\mathrm{y}^{1}=\mathrm{y}, \mathrm{y}(0)=1$, Exact Solution $\mathrm{y}=\mathrm{e}^{\mathrm{x}}, 0 \leq \mathrm{x} \leq 1$.

\begin{tabular}{|c|c|c|c|c|c|}
\hline $\mathbf{x}_{\mathbf{n}}$ & $\mathbf{h}$ & $\begin{array}{c}\text { Theoretical Solution } \\
\text { (Tsol) }\end{array}$ & $\begin{array}{c}\text { Numerical Solution } \\
\text { (Nsol) }\end{array}$ & $\begin{array}{c}\mathbf{N}_{\mathbf{f}} \\
\text { One-Step Rational Integrators of } \\
\text { order 5; k=3. Error(En) }\end{array}$ \\
\hline 0.1 & 0.1 & 1.10517092 & 1.261390920 & $-0.15622 \mathrm{E}-8$ & 1 \\
\hline 0.2 & 0.1 & 1.221400276 & 1.394050276 & $-0.17265 \mathrm{E}-8$ & 2 \\
\hline 0.3 & 0.1 & 1.34985881 & 1.540668810 & $-0.19081 \mathrm{E}-8$ & 3 \\
\hline 0.4 & 0.1 & 1.49182470 & 1.702707000 & $-0.23305 \mathrm{E}-8$ & 5 \\
\hline 0.5 & 0.1 & 1.64872127 & 1.882026270 & $-0.25756 \mathrm{E}-8$ & 6 \\
\hline 0.6 & 0.1 & 1.82211880 & 2.575782212 & $-0.28465 \mathrm{E}-8$ & 7 \\
\hline 0.7 & 0.1 & 2.01375271 & 2.298402710 & $-0.31459 \mathrm{E}-8$ & \\
\hline 0.8 & 0.1 & 2.22554093 & 2.540130930 & $-0.34768 \mathrm{E}-8$ & 8 \\
\hline 0.9 & 0.1 & 2.45960311 & 2.807283110 & $-0.38424 \mathrm{E}-8$ & 9 \\
\hline 1.0 & 0.1 & 2.71828183 & 3.102521830 & & 10 \\
\hline
\end{tabular}

Table 1: Efficiency of Our One- Step Rational Integrators Method of Order $2 k-1$ on Problem 1 for $H=0.1$

\begin{tabular}{|c|c|c|c|c|c|c|}
\hline \multirow[b]{2}{*}{ h } & \multirow[b]{2}{*}{$\begin{array}{c}\text { Theoretical } \\
\text { Solution }\end{array}$} & \multicolumn{3}{|c|}{ Aashikpelokhai (1991) } & \multirow{2}{*}{$\begin{array}{c}\text { Fatunla (1983) } \\
\text { Error x 106 } \\
\text { Order }=4\end{array}$} & \multirow[b]{2}{*}{$\mathbf{N}_{\mathbf{f}}$} \\
\hline & & $\begin{array}{l}\text { Error x 1012 } \\
\text { Order = 11 }\end{array}$ & $\begin{array}{c}\text { Error x } 10^{12} \\
\text { Order }=9\end{array}$ & $\begin{array}{c}\text { Error x } 10^{12} \\
\text { Order }=7\end{array}$ & & \\
\hline 0.1 & 1.10517092 & 0.00000 & 0.00000 & 0.00799 & 0.60450 & 1 \\
\hline 0.2 & 1.22140027 & 0.00022 & -0.00022 & 0.00866 & 0.48269 & 2 \\
\hline 0.3 & 1.34985881 & 0.00000 & 0.00000 & 0.00955 & 0.38781 & 3 \\
\hline 0.4 & 1.49182470 & -0.00022 & 0.00000 & 0.01066 & 0.36697 & 4 \\
\hline 0.5 & 1.64872127 & 0.00022 & 0.00000 & 0.01177 & 0.32105 & 5 \\
\hline 0.6 & 1.82211880 & -0.00022 & -0.00022 & 0.01288 & 0.02256 & 6 \\
\hline 0.7 & 2.01375271 & 0.00000 & 0.00000 & 0.01465 & 0.35937 & 7 \\
\hline 0.8 & 2.22554093 & -0.00044 & 0.00000 & 0.01554 & 0.34473 & 8 \\
\hline 0.9 & 2.45960311 & 0.00000 & 0.00000 & 0.01731 & 0.30697 & 9 \\
\hline 1.0 & 2.71828183 & 0.00044 & 0.00000 & 0.01954 & 0.03030 & 10 \\
\hline
\end{tabular}

Table 2: Error in Numerical Integrators with Uniform Mesh Size H=0.1 on Problem 1

From table 1, we observed that our One-Step rational integrators method of order 5 when compared with table 2 above shows that the One -step rational integrators method are efficient at resolving differential equations with exponential solutions. They compare favorably with Aashikpelokhai (1991) and Fatunla (1983).

Problem 3: Fatunla(1988) and Lambert(1978)

$y^{1}=1+y^{1} ; \mathrm{y}(0)=1 . \quad 0 \leq \mathrm{x} \leq 1 \quad$ Exact Solution $\mathrm{y}=\tan \left(\mathrm{x}+\frac{\pi}{4}\right)$ and $\mathrm{h}=0.05$

\begin{tabular}{|c|c|c|c|c|c|c|c|}
\hline $\mathbf{x}$ & $\mathbf{H}$ & $\begin{array}{c}\text { Theoretical } \\
\text { Solution }\end{array}$ & $\begin{array}{c}\text { Lambert and } \\
\text { Shaw }(1965 \\
\text { order } 4\end{array}$ & $\begin{array}{c}\text { Niekerk(1985) } \\
\text { order } 2\end{array}$ & $\begin{array}{c}\text { Fatunla(1988) } \\
\text { Order } 4\end{array}$ & $\begin{array}{l}\text { One-Step Rational } \\
\text { Integrators order } 5\end{array}$ & $\mathbf{N}_{\mathrm{f}}$ \\
\hline 0.10 & 0.05 & 1.22304888 & $9(-9)$ & $2(-6)$ & $6(-4)$ & $2(-10)$ & 1 \\
\hline 0.20 & 0.05 & 1.50849765 & $2(-7)$ & $8(-10)$ & $6(-5)$ & $2(-10)$ & 2 \\
\hline 0.30 & 0.05 & 1.89576512 & $4(-7)$ & $2(-10)$ & $5(-5)$ & $2(-10)$ & 3 \\
\hline 0.40 & 0.05 & 2.46491276 & $7(-7)$ & $5(-10)$ & $2(-4)$ & $2(-10)$ & 4 \\
\hline 0.50 & 0.05 & 3.40822234 & $1(-6)$ & $1(-10)$ & $2(-4)$ & $3(-10)$ & 5 \\
\hline 0.60 & 0.05 & 5.33185522 & $4(-6)$ & $5(-10)$ & $7(-4)$ & $5(-10)$ & 6 \\
\hline 0.65 & 0.05 & 7.34043658 & $8(-6)$ & $1(-10)$ & $1(-3)$ & $7(-10)$ & 7 \\
\hline 0.70 & 0.05 & 11.6813738 & $2(-2)$ & $3(-10)$ & $3(-3)$ & $1(-9)$ & 8 \\
\hline 0.75 & 0.05 & 28.2382529 & $1(-4)$ & $2(-10)$ & $1(-2)$ & $4(-9)$ & 9 \\
\hline
\end{tabular}

Table 3

Problem 4:Fatunla $(1980,1982)$

$y^{1}=\sqrt{\left(1-y^{2}\right)}, \mathrm{y}(0)=1,0 \leq x \geq \pi$, Exact Solution $\mathrm{y}=\operatorname{Cos}(\mathrm{x})$ and $\mathrm{h}=\frac{\pi}{10}$ 


\begin{tabular}{|c|c|c|c|c|c|c|c|}
\hline \multirow{3}{*}{$\mathbf{H}$} & \multirow{3}{*}{$\begin{array}{l}\text { Theoretical } \\
\text { Solution }\end{array}$} & \multicolumn{2}{|c|}{ Fatunla } & \multirow{2}{*}{\multicolumn{2}{|c|}{ Aashikpelokhai }} & \multirow{3}{*}{$\begin{array}{l}\text { One-Step Rational } \\
\text { Integrators order } 5\end{array}$} & \multirow{3}{*}{$\mathbf{N}_{\mathbf{f}}$} \\
\hline & & (1980) & (1982) & & & & \\
\hline & & Order 4 & Order 10 & Order 3 & Order 7 & & \\
\hline$\frac{\pi}{10}$ & 0.951056516 & 0.00000 & 0.0000 & -0.91617 & 0.09686 & 0.19780 & 1 \\
\hline$\frac{2 \pi}{10}$ & 0.809016994 & 0.13767 & 0.20110 & -0.71639 & 0.08607 & 0.22292 & 2 \\
\hline$\frac{3 \pi}{10}$ & 0.587785252 & 0.36666 & 0.50407 & -1.17123 & 0.06105 & 0.30635 & 3 \\
\hline$\frac{4 \pi}{10}$ & 0.309016994 & 0.42474 & 0.80596 & -0.64517 & 0.03582 & 0.48789 & 4 \\
\hline$\frac{5 \pi}{10}$ & 0.612574227 & 0.00152 & 1.39152 & -0.25006 & 0.01531 & 0.95893 & 5 \\
\hline$\frac{6 \pi}{10}$ & -0.309016994 & 0.28291 & 0.69429 & 0.05819 & -0.00183 & 5.81880 & 6 \\
\hline$\frac{7 \pi}{10}$ & -0.587785252 & 0.00411 & 0.59572 & 0.34945 & -0.01860 & -1.39682 & 7 \\
\hline$\frac{8 \pi}{10}$ & -0.809016994 & 0.32607 & 0.32176 & 0.68814 & -0.03795 & -0.36124 & 8 \\
\hline$\frac{9 \pi}{10}$ & -0.951056516 & 0.03518 & 0.03729 & 1.12100 & -0.06133 & -0.32195 & 9 \\
\hline$\Pi$ & 1.000000000 & 0.29774 & 0.0000 & 1.61343 & -0.08512 & -0.22508 & 10 \\
\hline
\end{tabular}

Our One-step rational integrators compare favourably with Fatunla $(1980,1982)$ and Aashikpelokhai (1991) in the solution of initial value problems (ivps) with oscillatory solution.

Problem 5: Modeling Advertising Awareness

$\mathrm{y}^{\wedge} 1=\mathrm{y}(1-\mathrm{y}), \quad \mathrm{y}(0)=1, \quad 0 \leq \mathrm{x} \leq 1 ;$ Exact Solution $\mathrm{y}=1-\mathrm{e}^{\wedge}(-0.693 \mathrm{x}), \quad \mathrm{h}=0.05$

\begin{tabular}{|c|c|c|c|c|c|c|c|}
\hline & & & & & & $\begin{array}{c}\text { Anetor and } \\
\text { Ebhohimen } \\
\text { (2013) }\end{array}$ & \\
\hline $\mathbf{X}$ & $\mathbf{h}$ & $\begin{array}{c}\text { Theorical } \\
\text { Solution }\end{array}$ & $\begin{array}{c}\text { Numerical } \\
\text { Solution }\end{array}$ & Error & $\begin{array}{c}\text { Fatunla } \\
\text { (1983) }\end{array}$ & Order 11 & $\mathbf{N}_{\mathbf{f}}$ \\
\hline & & & & (Order 5) & Order $=4$ & & \\
\hline 0.1 & 0.05 & 0.800029 & I.404529433 & $6.05 \mathrm{E}-13$ & $6.05 \mathrm{E}-07$ & $-1.39 \mathrm{E}-10$ & 1 \\
\hline 0.2 & 0.05 & 0.899985 & 1.382785 & $4.83 \mathrm{E}-13$ & $4.83 \mathrm{E}-07$ & $1.80 \mathrm{E}-10$ & 2 \\
\hline 0.3 & 0.05 & 0.849978 & 1.237788 & $3.88 \mathrm{E}-13$ & $3.88 \mathrm{E}-07$ & $3.05 \mathrm{E}-10$ & 3 \\
\hline 0.4 & 0.05 & 0.799971 & 1.166941 & $3.67 \mathrm{E}-13$ & $3.67 \mathrm{E}-07$ & $1.01 \mathrm{E}-10$ & 4 \\
\hline 0.5 & 0.05 & 0.749963 & 1.071013 & $3.21 \mathrm{E}-13$ & $3.21 \mathrm{E}-07$ & $1.53 \mathrm{E}-10$ & 5 \\
\hline 0.6 & 0.05 & 0.699956 & 0.722516 & $2.26 \mathrm{E}-14$ & $2.26 \mathrm{E}-08$ & $1.43 \mathrm{E}-10$ & 6 \\
\hline 0.7 & 0.05 & 0.649948 & 1.009318 & $3.59 \mathrm{E}-13$ & $3.59 \mathrm{E}-07$ & $9.57 \mathrm{E}-10$ & 7 \\
\hline 0.8 & 0.05 & 0.599941 & 0.944671 & $3.45 \mathrm{E}-13$ & $3.45 \mathrm{E}-07$ & $5.00 \mathrm{E}-10$ & 8 \\
\hline 0.9 & 0.05 & 0.549934 & 0.856904 & $3.07 \mathrm{E}-13$ & $3.07 \mathrm{E}-07$ & $2.16 \mathrm{E}-10$ & 9 \\
\hline 1 & 0.05 & 0.499926 & 0.530226 & $3.03 \mathrm{E}-14$ & $3.03 \mathrm{E}-08$ & $8.09 \mathrm{E}-10$ & 10 \\
\hline
\end{tabular}

Table 5

From the table above, we observed that our one - step rational integrator method of order 5 are efficient and also cope favorably when compared with Fatunla (1983) and Anetor and Ebhohimen 2013.

\section{Conclusion}

The implementations of the One-step rational integrators methodwere coded in Mathlab and run on a digital computer.

In conclusion, from the above results in tables 1,2,3,4 and 5 can be seen that our One-step rational integrators method of order five is efficient and accurate when compared with the existing methods of Aashikpelokhai 1991;Fatunla1983; Lambert and Shaw 1965 and Nieker 1985; Anetor and Ebhohimen 2013which can solve the same set of numerical initial value problems. The Region of Absolute Stability (RAS) of the One-step rational integrators method lies entirely on the Left-half of the complex plane. We therefore conclude that the One-step rational integrator methods are both A-stable and L-stable for $\mathrm{K}=3$. Hence, it is recommended for users, who are currently working in the area of research. 


\section{References}

i. Aashikpelokhai, U.S.U (1991): 'A Class of non-linear one- step rational integrator', Pon Publishers LimitedAgbor, Delta State.

ii. Aashikpelokhai U.S.U (2014): Renaissance of Mathematics in our Time. Pub. FNS, AAU Ekpoma.

iii. Aghajani and Moradifan, (2007): Oscillatory of solution of second order non-linear differential equations of Euler Type J. Mathematical Analysis and Applications 326, 1076 - 1089.

iv. Anetor 0. and EbhohimenF. (2013). Rational Interpolation Method for Solving Initial Value

v. Problem (IVPs) in Ordinary Differential Equations. International journal of Scientific and Research Publications, Volume 3, Issue 9, September, 2013. ISSN 2250-3153.

vi. Ebhohimen F. and Anetor 0 (2017); The Stability of the Rational Interpolation Method in

vii. Ordinary Differential Equations at $\mathrm{k}=6$. Transactions of the Nigerian Association of Mathematical physics Volume 5, (September and November, (2017); pp 33-38.

viii. ElakheO. A. and Aashikpelokhai, U.S.U. (2013) In: On A Singulo-Stiff Rational Integrator. International Journal of Natural and Applied Science, Vol. 2, No.1

ix. Fatunla, S.O. (1978); 'An implicit Two-part numerical integration Formula for linear and non-linear stiff system of ODE', Mathematics of Computation 32,1-11.

x. Fatunla,S.O.(1980,1982): 'Numerical integrators for stiff and highly oscillatory Differential equation'. Mathematics of Computation 34,373-390.

xi. Fatunla,S.O. (1988); 'Numerical methods for initial value problems in Ordinary Differential Equations'. Academic Press, IN INC USA.

xii. Lambert J.D and Shaw B. (1965): 'On the Numerical Solution of $y^{\prime}=f(x, y)$ by a class of formulae based on Rational Approximation'. Mathematics of Computation19:456-462

xiii. Lambert J.D. (1976) 'Two unconventional class of methods for stiff System'. (Ed-Willoughby, R.A.) Wildbald, W. Germany, 171-186.

xiv. Lambert J.D. (1978) 'Numerical Methods for Ordinary Differential Systems, John Wiley and Son, Chichester'.

xv. Lambert J.D. (1973); 'Computational Methods in ODE's John Wiley of sons, New York.

xvi. Obodi, (2001): Application of anseir epidemic model with delay, ABACUS 28(2) Maths Series, Sept, 2011.

xvii. Onianwa, C.U. and Aashikpelokhai,U.S.U. (2010): On the coefficient distribution of the stability Function of a High Order Integrator. J. of Maths Science 21(3) 233-244.

xviii. Ukpebor L.A and Aashikpelokhai, U.S.U. (2014): An Order 19 Rational Integrator. J. of NAMP Volume 28, No. 2, pp 167-174. 